Numer. Math. 74: 385-395

Numerische Mathematik

Electronic Edition

\title{
Error estimators for a mixed method
}

\author{
A. Alonso \\ Departamento de Matemática, Facultad de Ciencias Exactas, Universidad Nacional de La Plata, CC \\ 172, 1900 La Plata, Argentina
}

Received January 3, 1994 / Revised version received November 18, 1994

Summary. We present two a-posteriori error estimators for elliptic partial differential equations, when we use a mixed method. One is based on a adequate evaluation of the residual of the finite element solution and the other on the solution of a local problem. We prove their equivalence with the norm of the error, when the data is locally smooth.

\section{Introduction}

Several a posteriori error estimators have been introduced for the approximation by finite element methods of second order elliptic problems. Many of them are defined by evaluating in some way the residual of the finite element solutions, or by solving local problems for the error ([4], [1], [11], [2],[3], [12], [13]).

In many applications it is convenient to use mixed methods, which approximates simultaneously the original scalar variable and its gradient. Many finite element spaces have been introduced for this case, such as those of RaviartThomas [10] and Brezzi-Douglas-Marini [6], for example. Error estimators for mixed finite element methods have been introduced and analyzed in [11], [5], for the Stokes equations. Although the structure of that problem and the mixed formulation of second order scalar problems is the same, a straightforward extension of the techniques developed for the Stokes problem does not work in this case. Moreover, for the equation treated here it is possible to estimate the error for the vector variable (usually the most important) independently of the scalar one [9]. For this reason it is interesting to look for estimators only for the error of the vectorial variable.

In this paper we define estimators for the Raviart-Thomas and BrezziDouglas-Marini spaces (Sect.2). The first type of estimators (Sect. 3) is based on the computation of adequate norms of the residual. We prove its equivalence with the error, under some conditions of local regularity of the data. In Sect. 4, 
for the Raviart-Thomas space of lowest degree, we define an estimator, which it is based on the solution of a local problem. By assuming that the solution in the Brezzi-Douglas-Marini space of degree one approximates the exact solution better than that in the Raviart-Thomas space, we prove that this estimator is equivalent to the error.

\section{Description of mixed method and finite element spaces}

Let $\Omega$ be a bounded and simply connected polygon in $\mathbb{R}^{2}$ and let $\mathrm{u}$ be the solution of the problem

$$
\left\{\begin{aligned}
-\Delta u=\mathrm{f} & \text { in } \Omega \\
u=0 & \text { on } \partial \Omega
\end{aligned}\right.
$$

In many applications [7], the variable of interest is $\sigma=-\nabla u$ and for that reason it is reasonable to use a mixed finite element method which approximates $\sigma$ and u. The problem (2.1) is decomposed into a first order system:

$$
\left\{\begin{aligned}
\sigma+\nabla u=0 & \text { in } \Omega \\
\operatorname{div} \sigma=\mathrm{f} & \text { in } \Omega \\
u=0 & \text { on } \partial \Omega
\end{aligned}\right.
$$

and the correspondent weak formulation is

$$
\left\{\begin{array}{rlrl}
(\boldsymbol{\sigma}, \boldsymbol{\tau})-(\operatorname{div} \boldsymbol{\tau}, u) & =0 & & \forall \boldsymbol{\tau} \in H(\operatorname{div}, \Omega) \\
(\operatorname{div} \boldsymbol{\sigma}, v) & =(f, v) & \forall v \in L^{2}(\Omega)
\end{array}\right.
$$

where ( , ) denotes the $L^{2}$-product, and

$$
H(\operatorname{div}, \Omega)=\left\{\boldsymbol{\tau} \in\left(L^{2}(\Omega)\right)^{2}: \operatorname{div} \boldsymbol{\tau} \in L^{2}(\Omega)\right\}
$$

We shall use the standard notation for the Sobolev spaces $H^{m}(D)$, their norms \|\|$_{m, D}$ and seminorms ||$_{m, D}$.

Let $\mathscr{T}$ be a regular family of triangulations of $\Omega$, as usual $h$ stands for the maximum meshsize; i.e., there exists a minimum angle smaller than all the angles of all the triangles of all the meshes.

We shall use $c, c_{1}, c_{2}$, etc. to denote generic constants not necessarily the same at each ocurrence. In general these constants only will depend on the minimum angle but not on the meshsize $h$.

The Raviart-Thomas spaces [10] are defined for $k \geq 0$ by

$R T^{k}=V_{h}^{k} \times W_{h}^{k}$, where

$V_{h}^{k}=\left\{\boldsymbol{\tau} \in H(\operatorname{div}, \Omega):\left.\boldsymbol{\tau}\right|_{T} \in \mathbf{P}_{\mathbf{k}}(T)+\mathbf{x} P_{k}(T)\right\}$,

$W_{h}^{k}=\left\{v \in L^{2}(\Omega):\left.v\right|_{T} \in P_{k}(T)\right\}$

and where $P_{k}(T)$ denotes the spaces of polynomial of degree less than or equal to $k$ and $\mathbf{P}_{\mathbf{k}}(T)=\left(P_{k}(T)\right)^{2}$

The Brezzi-Douglas-Marini spaces [6] are defined for $k \geq 1$ by 


$$
\begin{aligned}
& B D M^{k}=V_{h}^{k} \times W_{h}^{k}, \text { where } \\
& V_{h}^{k}=\left\{\boldsymbol{\tau} \in H(\operatorname{div}, \Omega):\left.\boldsymbol{\tau}\right|_{T} \in \mathbf{P}_{\mathbf{k}}(T)\right\}, \\
& W_{h}^{k}=\left\{v \in L^{2}(\Omega):\left.v\right|_{T} \in P_{k-1}(T)\right\} .
\end{aligned}
$$

The discrete problem is then given by: Find $\left(\sigma_{h}, u_{h}\right) \in V_{h}^{k} \times W_{h}^{k}$ such that

$$
\left\{\begin{array}{rlrl}
\left(\boldsymbol{\sigma}_{h}, \boldsymbol{\tau}\right)-\left(\operatorname{div} \boldsymbol{\tau}, u_{h}\right) & =0 & & \forall \boldsymbol{\tau} \in V_{h}^{k} \\
\left(\operatorname{div} \boldsymbol{\sigma}_{h}, v\right) & =(f, v) & \forall v \in W_{h}^{k}
\end{array}\right.
$$

Let $\boldsymbol{\epsilon}_{h}=\boldsymbol{\sigma}-\boldsymbol{\sigma}_{h}$ denote the error of the vector variable.

Let $\boldsymbol{\tau} \in H(\operatorname{div}, \Omega)$ such that $\operatorname{div} \boldsymbol{\tau}=0$. Then $\boldsymbol{\tau}=\operatorname{curl} \varphi$, with $\varphi \in H^{1}(\Omega)$. In this case, from (2.3) we obtain

$$
\left(\epsilon_{h}, \boldsymbol{\tau}\right)=-\left(\sigma_{h}, \operatorname{curl} \varphi\right)=\sum_{T \in \mathscr{T} h}\left[-\left(\operatorname{rot} \sigma_{h}, \varphi\right)_{T}-\int_{\partial T} \varphi \sigma_{h} \cdot t\right]
$$

where for each triangle $\mathrm{T}, \mathrm{t}$ is its unit tangent vector.

For each interior edge $l$ of the triangulation. $\mathscr{T}_{h}$, let $\llbracket \sigma_{h} . t \rrbracket_{l}$ denote the jump of $\sigma_{h} . t$ across the edge $l$.

Let $J_{l}=\left\{\begin{array}{cc}\llbracket \sigma_{h} . t \rrbracket_{l} & \text { if } l \not \subset \partial \Omega \\ 2\left(\boldsymbol{\sigma}_{h} . t\right) & \text { if } l \subset \partial \Omega\end{array}\right.$

Whit this notation we may write the residual equation:

For $\boldsymbol{\tau}=\operatorname{curl} \varphi \in H(\operatorname{div}, \Omega)$

$$
\left(\epsilon_{h}, \tau\right)=\sum_{T \in \mathscr{T}_{h}}\left[-\left(\operatorname{rot} \sigma_{h}, \varphi\right)_{T}-\frac{1}{2} \sum_{l \subset \partial T} \int_{l} J_{l} \varphi\right]
$$

Given an integer $k$, let $P_{h}^{k}$ be the $L^{2}$-projection onto $W_{h}^{k}$. Since $\operatorname{div} \sigma_{h} \in$ $W_{h}^{k}$, from (2.3) and (2.4), we see that

$$
\operatorname{div} \boldsymbol{\epsilon}_{h}=f-P_{h} f, \text { for } \sigma_{h} \in R T^{k} \text { or } \boldsymbol{\sigma}_{h} \in B D M^{k+1}
$$

\section{Error estimators based on the residual}

First we work with the $R T^{k}$-spaces, $k \geq 0$.

For any $T \in \mathscr{T}$, we define

$$
\eta_{T}^{2}=|T|\left\|\operatorname{rot} \sigma_{h}\right\|_{0, T}^{2}+\frac{1}{2} \sum_{l \subset \partial T}|l|\left\|J_{l}\right\|_{0, l}^{2}
$$

where $|T|$ and $|l|$ are the area of $\mathrm{T}$ and the length of $l$, resp. and let

$$
\eta=\left(\sum_{T \in \mathscr{T} h} \eta_{T}^{2}\right)^{1}
$$


Theorem 3.1 There exist two positive constants $c_{1}$ and $c_{2}$, only depending on the minimum angle of the mesh such that

$$
\begin{gathered}
\left\|\boldsymbol{\epsilon}_{h}\right\|_{0, \Omega} \leq c_{1}\left[\sum_{T \in \mathscr{T}}\left(\eta_{T}^{2}+|T|\left\|f-P_{h}^{k} f\right\|_{0, T}^{2}\right)\right]^{\frac{1}{2}} \\
\eta \leq c_{2}\left\|\boldsymbol{\epsilon}_{h}\right\|_{0, \Omega}
\end{gathered}
$$

Proof. We first prove the upper bound (3.3). Since $\epsilon_{h} \in L^{2}(\Omega)$, we may decompose it as $\epsilon_{h}=\nabla p+\operatorname{curl} \varphi$, where $p \in H_{0}^{1}(\Omega)$ and $\varphi \in H^{1}(\Omega)$. Then

$\left\|\epsilon_{h}\right\|_{0, \Omega}^{2}=\left(\epsilon_{h}, \nabla p\right)+\left(\epsilon_{h}, \operatorname{curl} \varphi\right)$

Since $p \in H_{0}^{1}(\Omega), P_{h}^{k} f \in W_{h}^{k}$, using (2.6), we get

$$
\begin{aligned}
\left(\epsilon_{h}, \nabla p\right) & =-\left(\operatorname{div} \epsilon_{h}, p\right)=-\sum_{T \in \mathscr{T}_{h}}\left(f-P_{h}^{k} f, p-P_{h}^{k} p\right)_{T} \\
& \leq\left. c \sum_{T \in \mathscr{T}}|T|^{2}\left|f-P_{h}^{k} f \|_{0, T}\right| p\right|_{1, T}
\end{aligned}
$$

Let $\varphi^{\mathrm{I}} \in \mathscr{D}_{k+1}^{1}(\Omega)=\left\{w \in H^{1}(\Omega):\left.w\right|_{T} \in P_{k+1}(T)\right\}$ be an interpolant of $\varphi$ such that

$$
\begin{array}{ll}
\left\|\varphi-\varphi^{\mathrm{I}}\right\|_{0, T} \leq c|T|^{\frac{1}{2}}|\varphi|_{1, \tilde{T}} & \forall T \in \mathscr{T} \\
\left\|\varphi-\varphi^{\mathrm{I}}\right\|_{0, l} \leq c|l|^{2}|\varphi|_{1, \tilde{T}} & \forall l \subset \partial T
\end{array}
$$

where $\tilde{T}=\bigcup\left\{T^{*} \in \mathscr{T}_{h}: T\right.$ and $T^{*}$ have a common vertex $\}$

( $\varphi^{\mathrm{I}}$ may be, for example, the Clement-interpolation [8].)

Since $\operatorname{curl} \varphi^{\mathrm{I}} \in V_{h}^{k}$, then $\left(\epsilon_{h}, \operatorname{curl} \varphi^{\mathrm{I}}\right)=0$. By using (2.5), and (3.6), we may write

$$
\begin{aligned}
\left(\epsilon_{h}, \operatorname{curl} \varphi\right) & =\left(\epsilon_{h}, \operatorname{curl}\left(\varphi-\varphi^{\mathrm{I}}\right)\right) \\
& =\sum_{T \in \mathscr{T} h}\left[-\left(\operatorname{rot} \sigma_{h}, \varphi-\varphi^{\mathrm{I}}\right)_{T}-\frac{1}{2} \sum_{l \subset \partial T} \int_{l} J_{l}\left(\varphi-\varphi^{\mathrm{I}}\right)\right] \\
& \leq \sum_{T \in \mathscr{T}}\left[c\left\|\operatorname{rot} \sigma_{h}\right\|_{0, T}|T|^{\frac{1}{2}}|\varphi|_{1, \tilde{T}}+\frac{1}{2} \sum_{l \subset \partial T} c\left\|J_{l}\right\|_{0, l}|l|^{\frac{1}{2}}|\varphi|_{1, \tilde{T}}\right]
\end{aligned}
$$

Since $|p|_{1, T} \leq\left\|\boldsymbol{\epsilon}_{h}\right\|_{0, T},|\varphi|_{1, T} \leq\left\|\boldsymbol{\epsilon}_{h}\right\|_{0, T}$ and the number of triangles in $\tilde{T}$ only depends on the minimum angle of $\mathscr{T}$, by using (3.5) and (3.7) we arrive at the desired estimate (3.3).

In order to proof (3.4) we use the following lemma:

Lemma 3.1 Let $T \in \mathscr{T}$. Given $q_{T} \in L^{2}(T), p_{l, T} \in L^{2}(l), l \subset \partial T$, then $\exists ! \psi_{T} \in$ $P_{k+3}(T)$ such that

$$
\begin{cases}\left(\psi_{T}, r\right)_{T}=\left(q_{T}, r\right)_{T} & \forall r \in P_{k}(T) \\ \int_{l} \psi_{T} s=\int_{l} p_{l, T} S & \forall s \in P_{k+1}(l) \\ \psi_{T}=0 & \text { at the vertices of } T\end{cases}
$$




$$
\left\|\psi_{T}\right\|_{0, T} \leq c\left(\left\|q_{T}\right\|_{0, T}+\sum_{l \subset \partial T}|l|^{\frac{1}{2}}\left\|p_{l, T}\right\|_{0, l}\right)
$$

where $c$ only dependends on the minimum angle.

Proof. Clearly (3.8) is a square linear system with $(k+1)(k+2) / 2+3(k+2)+3$ equations and unknowns. Easily we prove its uniqueness by considering the case $q_{T}=0$ and $p_{l, T}=0 l \subset \partial T$. Moreover, we also prove that if $\int_{l} \psi_{T} s=0$ $\forall s \in P_{k+1}(l)$, then $\left.\psi_{T}\right|_{l}=0$. Finally (3.9) follows by standard homogeneity arguments.

We apply the lemma for $q_{T}=-|T| \operatorname{rot} \sigma_{h} \in P_{k}(T)$ and $p_{l, T}=-|l| J_{l} \in$ $P_{k+1}(l)$ in each $T \in \mathscr{T}_{h}$. Let us define $\psi$ such that $\left.\psi\right|_{T}=\psi_{T}$. Since $\psi$ is piecewise polynomial and continuous, then $\psi \in H^{1}(\Omega)$. By using (3.9) and a inverse inequality

$$
\|\operatorname{curl} \psi\|_{0, T} \leq c\left(|T|^{\frac{1}{2}}\left\|\operatorname{rot} \boldsymbol{\sigma}_{h h}\right\|_{0, T}+\sum_{l \in \partial T}|l|^{\frac{1}{2}}\left\|J_{l}\right\|_{0, l}\right)
$$

This, in conjunction with (3.8) and the residual equation (2.5),yields

$$
\begin{gathered}
\eta^{2}=\sum_{T \in \mathscr{T}}\left[|T|\left\|\operatorname{rot} \boldsymbol{\sigma}_{h}\right\|_{0, T}^{2}+{ }_{2}^{1} \sum_{l \subset \partial T}|l|\left\|J_{l}\right\|_{0, l}^{2}\right]=\sum_{T \in \mathscr{T}_{h}}\left[-\left(\operatorname{rot} \boldsymbol{\sigma}_{h}, \psi\right)_{T}-\right. \\
\left.-\frac{1}{2} \sum_{l \subset \partial T} \int_{l} J_{l} \psi\right]=\sum_{T \in \mathscr{T}_{h}}\left(\boldsymbol{\epsilon}_{h}, \operatorname{curl} \psi\right)_{T} \leq \sum_{T \in \mathscr{T}}\left\|\boldsymbol{\epsilon}_{h}\right\|_{0, T}\|\operatorname{curl} \psi\|_{0, T} \leq \\
\leq c\left(\sum_{T \in \mathscr{T}}\left(|T|\left\|\operatorname{rot} \boldsymbol{\sigma}_{h}\right\|_{0, T}^{2}+\frac{1}{2} \sum_{l \subset \partial T}|l|\left\|J_{l}\right\|_{0, l}^{2}\right)\right)^{\frac{1}{2}}\left\|\boldsymbol{\epsilon}_{h}\right\|_{0, \Omega} \leq c \eta\left\|\boldsymbol{\epsilon}_{h}\right\|_{0, \Omega}
\end{gathered}
$$

This completes the proof.

When the data $f$ is locally smooth, then the estimator is equivalent up to higher order term to the error. In fact, we have the following theorem.

Theorem 3.2 Let us assume that $\left.f\right|_{T} \in H^{k+1}(T) \quad \forall T \in \mathscr{T}_{h}$. Then there exists a positive constant $c$ such that

$$
\left\|\boldsymbol{\epsilon}_{h}\right\|_{0, \Omega} \leq c\left(\eta+h^{k+2}\left(\sum_{T \in \mathscr{T}}|f|_{k+1, T}^{2}\right)^{\frac{1}{2}}\right)
$$

Proof. By using the approximation properties of $P_{h}^{k}$, by (3.3) we obtain (3.11). 
Remark 3.1 Let us assume that there exists a triangulation $\mathscr{T}$ such that $\left.f\right|_{T} \in$ $H^{k+1}(T) \forall T \in \mathscr{T}$, and let us also assume that all the triangulations $\mathscr{T} h$ are refinements of $\mathscr{T}$. If there exists a constant $c>0$ not depending on $h$ such that $\left\|\boldsymbol{\epsilon}_{h}\right\|_{0, \Omega} \geq c h^{k+1}$, then for $h \leq h^{*}$, the estimator is equivalent to the error.

Let us now consider the spaces $B D M^{k+1}, k \geq 0$

For any $T \in \mathscr{T}$, we define

$$
\begin{aligned}
& \eta_{T}^{2}=|T|\left\|\operatorname{rot} \sigma_{h}\right\|_{0, T}^{2}+\frac{1}{2} \sum_{l \subset \partial T}|l|\left\|J_{l}\right\|_{0, l}^{2}+|T|\left\|f-P_{h}^{k} f\right\|_{0, T}^{2} \\
& \eta=\left(\sum_{T \in \mathscr{T}} \eta_{T}^{2}\right)^{\frac{1}{2}}
\end{aligned}
$$

Theorem 3.3 There exist two constants $c_{1}, c_{2}$ only depending on the regularity of the mesh such that

$$
\begin{gathered}
\left\|\epsilon_{h}\right\|_{0, \Omega} \leq c_{1} \eta \\
\eta \leq c_{2}\left(\left\|\epsilon_{h}\right\|_{0, \Omega}+\left(\sum_{T \in \mathscr{T}}|T|\left\|f-P_{h}^{k+1} f\right\|_{0, T}^{2}\right)^{\frac{1}{2}}\right)
\end{gathered}
$$

Moreover, iff $\left.\right|_{T} \in H^{k+2}(T), \forall T \in \mathscr{T}$, then there exists a positive constant c such that

$$
\eta \leq c\left(\left\|\boldsymbol{\epsilon}_{h}\right\|_{0, \Omega}+h^{k+3}\left(\sum_{T \in \mathscr{T}}|f|_{k+2, T}^{2}\right)^{\frac{1}{2}}\right)
$$

Proof. The proof of (3.13) is identical to (3.3).

By calling $\eta_{1, T}^{2}=|T|\left\|\operatorname{rot} \sigma_{h}\right\|_{0, T}^{2}+\sum_{l \subset \partial T}|l|\left\|J_{l}\right\|_{0, l}^{2}$ and by repeating the proof of (3.4) we obtain

$$
\sum_{T \in \mathscr{T}} \eta_{1, T}^{2} \leq c\left\|\boldsymbol{\epsilon}_{h}\right\|_{0, \Omega}^{2}
$$

It remains only to analyze the term $\eta_{2, T}^{2}=|T|\left\|f-P_{h}^{k} f\right\|_{0, T}^{2}$.

Let $B_{k+4}(T)=\left\{p \in P_{k+4}(T): p=0\right.$ on $\left.\partial T\right\}$. We use the following lemma:

Lemma 3.2 Let $T \in \mathscr{T}$. Given $g \in L^{2}(T)$, then $\exists ! p \in B_{k+4}(T)$ such that

$$
\begin{array}{ll}
(p, r)_{T}=(g, r)_{T} & \forall r \in P_{k+1}(T) \\
\|p\|_{0, T} \leq c\|g\|_{0, T} & c \text { independ. of } h
\end{array}
$$


For each $T \in \mathscr{T}$, we select $g=|T|\left(f-P_{h}^{k} f\right)$. Since $|p|_{1, T} \leq c h^{-1}\|p\|_{0, T}$, by using (3.17) we arrive at

$$
\begin{gathered}
\|p\|_{0, T} \leq c|T|^{\frac{1}{2}} \eta_{2, T} \\
|p|_{1, T} \leq c \eta_{2, T}
\end{gathered}
$$

Since $p \in H_{0}^{1}(\Omega), P_{h}^{k+1} f-P_{h}^{k} f \in P_{k+1}(T)$ by using (2.6) we may write

$$
\begin{gathered}
\sum_{T \in \mathscr{T}_{h}} \eta_{2, T}^{2}=\sum_{T \in \mathscr{T} h}\left[|T|\left(f-P_{h}^{k} f, f-P_{h}^{k+1} f\right)_{T}+|T|\left(f-P_{h}^{k} f, P_{h}^{k+1} f-P_{h}^{k} f\right)_{T}\right] \\
=\sum_{T \in \mathscr{T}}\left[|T|\left(f-P_{h}^{k} f, f-P_{h}^{k+1} f\right)_{T}+\left(p, P_{h}^{k+1} f-P_{h}^{k} f\right)_{T}\right] \\
=\sum_{T \in \mathscr{T}}\left[|T|\left(f-P_{h}^{k} f, f-P_{h}^{k+1} f\right)_{T}+\left(p, f-P_{h}^{k} f\right)_{T}+\left(p, P_{h}^{k+1} f-f\right)_{T}\right] \\
=\sum_{T \in \mathscr{T}}\left[|T|\left(f-P_{h}^{k} f, f-P_{h}^{k+1} f\right)_{T}-\left(\nabla p, \boldsymbol{\epsilon}_{h}\right)_{T}+\left(p, P_{h}^{k+1} f-f\right)_{T}\right] \\
\leq c \sum_{T \in \mathscr{T} h}\left[\eta_{2, T}|T|^{1}|| f-P_{h}^{k+1}\left\|_{0, T}+\eta_{2, T}\right\| \epsilon_{h}\left\|_{0, T}+\eta_{2, T}|T|^{2}\right\| f-P^{k+1} f \|_{0, T}\right]
\end{gathered}
$$

Hence

$$
\sum_{T \in \mathscr{T} h} \eta_{2, T}^{2} \leq c\left[\left\|\epsilon_{h}\right\|_{0, \Omega}^{2}+\sum_{T \in \mathscr{T} h}|T|\left\|f-P_{h}^{k+1} f\right\|_{0, T}^{2}\right]
$$

By (3.16) and (3.18) we obtain (3.14).

Finally, by proceeding as in Theorem (2.2) we prove (3.15).

Remark 3.2 If there exists $c>0$ not depending on h such that $\left\|\boldsymbol{\epsilon}_{h}\right\|_{0, \Omega} \geq c h^{k+2}$, then, analogously to the case of the $R T^{k}$-spaces, the estimator is equivalent to the error.

Remark 3.3 The lower bounds in (3.4) and (3.14) are indeed of a local character. In fact it could be proved that

$$
\eta_{T} \leq c\left(\sum_{T^{*} \in \tilde{T}}\left\|\boldsymbol{\epsilon}_{h}\right\|_{0, T^{*}}^{2}\right)^{\frac{1}{2}}
$$

To do this, Lemma 3.1 should be applied to a lightly choice of $q_{T}$ and $\mathrm{p}_{l, T}$. $\left(q_{T}=0\right.$ in $T^{*} \subset \tilde{T} / T$ and $\mathrm{p}_{l, T}=0$ on $\left.l \subset \tilde{T} / T\right)$

(3.19) shows that the estimator $\eta_{T}$ can be used to determine the triangles to be refined since a big local estimator $\eta_{T}$ implies a big error in $\tilde{T}$. 


\section{An error estimator based on the solution of a local problem}

For the space $R T^{0}$, it is simply to define a second estimator from a local problem, which has the same structure as (2.5). With this purpose we define an adequate discrete space. For the special case of the space $R T^{0}$, $\operatorname{rot} \sigma_{h}=0$, and (2.5) may be written as

$$
\left(\epsilon_{h}, \boldsymbol{\tau}\right)=\sum_{T \in \mathscr{T}}\left(-\frac{1}{2} \sum_{l \subset \partial T} \int_{l} J_{l} \varphi\right) \quad \text { for } \boldsymbol{\tau}=\operatorname{curl} \varphi \in H(\operatorname{div}, \Omega)
$$

Let $\Pi_{h}$ be the projection-operator onto $R T^{0}[10]$ such that, for $\tau \in H(\operatorname{div}, \Omega)$

$$
\left(P_{h}^{0}(\operatorname{div} \boldsymbol{\tau}), v\right)=\left(\operatorname{div} \Pi_{h} \boldsymbol{\tau}, v\right) \quad \forall v \in W_{h}^{0}
$$

that is

$$
(\operatorname{div} \boldsymbol{\tau}, v)_{T}=\left(\operatorname{div} \Pi_{h} \boldsymbol{\tau}, v\right)_{T} \quad \forall v \in P_{0}(T)
$$

It is known that [8],

$$
\left\|\boldsymbol{\tau}-\Pi_{h} \boldsymbol{\tau}\right\|_{0, T} \leq c h|\boldsymbol{\tau}|_{1, T} \quad \text { if }\left.\boldsymbol{\tau}\right|_{T} \in\left(H^{1}(T)\right)^{2}
$$

Let $S^{0}=\left\{\boldsymbol{\tau} \in B D M^{1}: \Pi_{h} \boldsymbol{\tau}=0\right\}$

In particular, for $\tau \in S^{0}$, since $\operatorname{div} \tau \in V_{h}^{0}$ we observe that $\operatorname{div} \boldsymbol{\tau}=0$. Let $P_{2}^{0}(T)=\left\{\varphi \in P_{2}(T): \varphi=0\right.$ at the vertices of $\left.\mathrm{T}\right\}$

Lemma 4.1 $S^{0}=\left\{\operatorname{curl} \varphi: \varphi \in H^{1}(\Omega):\left.\varphi\right|_{T} \in P_{2}^{0}(T)\right\}$

Proof. Let $\boldsymbol{\tau} \in S^{0}$. Since $\operatorname{div} \boldsymbol{\tau}=0$ there exists $\varphi \in H^{1}(\Omega)$ such that $\boldsymbol{\tau}=$ curl $\varphi$, and $\left.\varphi\right|_{T} \in P_{2}(T)$. For each edge $l \subset \partial T$, let $x_{l}$ denotes the midpoint of $l$, and $\eta$ its unit outer normal vector.

Since $\Pi_{h} \boldsymbol{\tau}=0$, then $\boldsymbol{\tau} . \eta\left(x_{l}\right)=0$. But $\boldsymbol{\tau} \cdot \eta\left(x_{l}\right)=\nabla \varphi . t\left(x_{l}\right)=0$.Hence, by choosing $\varphi$ such that it vanishs at one of the vertices of $\mathrm{T}$, it must vanish at all them.

On the other hand, if $\varphi \in H^{1}(\Omega):\left.\varphi\right|_{T} \in P_{2}^{0}(T)$, then $\boldsymbol{\tau}=\operatorname{curl} \varphi \in B D M^{1}$, and $\boldsymbol{\tau} . \eta\left(x_{l}\right)=0$. Then $\Pi_{h} \boldsymbol{\tau}=0$, and hence $\boldsymbol{\tau} \in S^{0}$.

Let $S_{T}=\left\{\operatorname{curl} \varphi: \varphi \in P_{2}^{0}(T)\right\}$.

We define the following discrete problem:

To find $\varphi_{T} \in P_{2}^{0}(T)$ such that

$$
\left(\operatorname{curl} \varphi_{T}, \operatorname{curl} \varphi\right)_{T}=-\frac{1}{2}\left(\sum_{l \subset \partial T} \int_{l} J_{l} \varphi\right) \forall \varphi \in P_{2}^{0}(T)
$$

There exists a unique solution $\varphi_{T}$. Let $e_{T}=\operatorname{curl} \varphi_{T}$. Then $e_{T} \in S_{T}$. We define

$$
e \in \prod_{T \in \mathscr{T} h} S_{T} \quad:\left.e\right|_{T}=e_{T}
$$

Let $\sigma_{2} \in B D M^{1}$ be the solution of (2.4). 
Let us suppose that $\sigma_{2}$ approximates better than $\sigma_{h}$, that is, there exists a positive constant $\gamma<1$ such that

$$
\left\|\boldsymbol{\sigma}_{2}-\boldsymbol{\sigma}\right\|_{0, \Omega} \leq \gamma\left\|\boldsymbol{\epsilon}_{h}\right\|_{0, \Omega}
$$

This condition can be seen as a saturation assumption in the sense of BankWeiser [4]; that is, we assume that a higher order method gives a better approximation than a lower order one. This is (assimptotically) the case when the solution $u \in H^{2+\epsilon}(\Omega) \quad(\epsilon>0)$. In practice, when reasonably refined meshes are used, (4.4) is always true.

Then we have the following theorem.

Theorem 4.1 Under assumption (4.4), then there exist two positive constants $c_{1}$ and $c_{2}$, only depending on the regularity of the mesh and on $\gamma$ such that, for $h \leq h^{*}$

$$
\begin{gathered}
\left\|\boldsymbol{\epsilon}_{h}\right\|_{0, \Omega} \leq c_{1}\|e\|_{0, \Omega} \\
\|e\|_{0, \Omega} \leq c_{2}\left\|\boldsymbol{\epsilon}_{h}\right\|_{0, \Omega}
\end{gathered}
$$

Proof. $\left\|\boldsymbol{\epsilon}_{h}\right\|_{0, \Omega} \leq\left\|\boldsymbol{\sigma}-\boldsymbol{\sigma}_{2}\right\|_{0, \Omega}+\left\|\boldsymbol{\sigma}_{2}-\boldsymbol{\sigma}_{h}\right\|_{0, \Omega}$.

Let $\epsilon_{2}=\sigma_{2}-\sigma_{h} \in B D M^{1}$. By subtracting (2.4) from (2.3), and by using (2.6), we obtain $\left(\sigma-\sigma_{2}, \epsilon_{2}\right)=0$

Moreover, since $\operatorname{div}\left(\Pi_{h}\left(\epsilon_{2}\right)\right)=P_{h}^{0}\left(\operatorname{div} \epsilon_{2}\right)=0$, then $\left(\epsilon_{h}, \Pi_{h} \epsilon_{2}\right)=0$.

$\epsilon_{2}-\Pi_{h} \epsilon_{2} \in S^{0}$, because $\epsilon_{2}-\Pi_{h} \epsilon_{2} \in B D M^{1}$ and $\Pi_{h}\left(\epsilon_{2}-\Pi_{h} \epsilon_{2}\right)=0$. By Lemma 4.1, there exists $\varphi \in H^{1}(\Omega),\left.\varphi\right|_{T} \in P_{2}^{0}(T)$ such that $\operatorname{curl} \varphi=\epsilon_{2}-\Pi_{h} \epsilon_{2}$. By using (4.1), (4.2) and (4.3) we may write

$$
\begin{aligned}
\left\|\epsilon_{2}\right\|_{0, \Omega}^{2} & =\left(\sigma_{2}-\sigma, \epsilon_{2}\right)+\left(\boldsymbol{\epsilon}_{h}, \boldsymbol{\epsilon}_{2}\right)=\left(\boldsymbol{\epsilon}_{h}, \boldsymbol{\epsilon}_{2}-\Pi_{h} \boldsymbol{\epsilon}_{2}\right) \\
& =\sum_{T \in \mathscr{T}}-\frac{1}{2} \sum_{l \subset \partial T} \int_{l} J_{l} \varphi=\sum_{T \in \mathscr{T}}\left(e_{T}, \boldsymbol{\epsilon}_{2}-\Pi_{h} \boldsymbol{\epsilon}_{2}\right)_{T} \\
& \leq \sum_{T \in \mathscr{T}}\left\|e_{T}\right\|_{0, T}\left\|\boldsymbol{\epsilon}_{2}-\Pi_{h} \boldsymbol{\epsilon}_{2}\right\|_{0, T} \leq \sum_{T \in \mathscr{T}} c\left\|e_{T}\right\|_{0, T} h\left|\boldsymbol{\epsilon}_{2}\right|_{1, T} \\
& \leq c \sum_{T \in \mathscr{T}}\left\|e_{T}\right\|_{0, T}\left\|\epsilon_{2}\right\|_{0, T} \leq c\|e\|_{0, \Omega}\left\|\boldsymbol{\epsilon}_{2}\right\|_{0, \Omega}
\end{aligned}
$$

Hence

$$
\left\|\boldsymbol{\epsilon}_{2}\right\|_{0, \Omega} \leq c\|e\|_{0, \Omega}
$$

By (4.4) and (4.7) we arrive at

$$
\left\|\boldsymbol{\epsilon}_{h}\right\|_{0, \Omega} \leq \gamma\left\|\boldsymbol{\epsilon}_{h}\right\|_{0, \Omega}+c\|e\|_{0, \Omega}
$$

and, since $\gamma<1$ we obtain the bound (4.5).

By the definition of the estimator (4.3)

$$
\sum_{T \in \mathscr{T}_{h}}\left\|e_{T}\right\|_{0, T}^{2}=\sum_{T \in \mathscr{T}_{h}}-\frac{1}{2}\left(\sum_{l \subset \partial T} \int_{l} \varphi_{T} J_{l}\right)
$$


For each edge $l \subset T_{1} \cap T_{2}$, let $\left[\varphi_{l}\right]_{A}=\frac{1}{2}\left(\varphi_{T_{1} / l}+\varphi_{T_{2} / l}\right)$-that is, the average across the edge -. $\left[\varphi_{l}\right]_{A} \in P_{2}^{0}(l)$. Then, we may re-write (4.8)

$$
\sum_{T \in \mathscr{T} h}\left\|e_{T}\right\|_{0, T}^{2}=\sum_{T \in \mathscr{T} h}-{ }_{2}^{1}\left(\sum_{l \subset \partial T} \int_{l}\left[\varphi_{l}\right]_{A} J_{l}\right)
$$

For each $l$, let $\psi_{l} \in H^{1}(\Omega)$ be the piecewise quadratic function such that $\psi_{l}\left(x_{l}\right)=1$ and vanishes at all nodes and remaining midpoints of $\mathscr{T}_{h}$, and we consider $\varphi=\sum_{l}\left[\varphi_{l}\right]_{A}\left(x_{l}\right) \psi_{l}$. Then $\varphi \in H^{1}(\Omega)$ and satisfies

$$
\int_{l} \varphi J_{l}=\int_{l}\left[\varphi_{l}\right]_{A} J_{l} \quad l \subset \partial T \quad \forall T \in \mathscr{T}_{h}
$$

Moreover, since $\varphi_{T_{i}} \in P_{2}^{0}\left(T_{i}\right)$,

$$
\begin{aligned}
\left|\left[\varphi_{l}\right]_{A}\left(x_{l}\right)\right| & \leq \frac{1}{2}\left(\left|\varphi_{T_{1}}\left(x_{l}\right)\right|+\left|\varphi_{T_{2}}\left(x_{l}\right)\right|\right) \\
& \leq c|l|^{-\frac{1}{2}}\left[\left\|\varphi_{T_{1}}\right\|_{0, \partial T_{1}}+\left\|\varphi_{T_{2}}\right\|_{0, \partial T_{2}}\right]
\end{aligned}
$$

and

$$
\left\|\varphi_{T}\right\|_{0, \partial T} \leq c h^{2}\left|\varphi_{T}\right|_{1, T}=c h^{2}\left\|e_{T}\right\|_{0, T} \quad \text { for each } T \in \mathscr{T}
$$

By using (4.10)-(4.12) and reminding that $\left\|\operatorname{curl} \psi_{l}\right\|_{0, T} \leq c$, we obtain

$$
\begin{aligned}
\|\operatorname{curl} \varphi\|_{0, T} & \leq \sum_{l \subset \partial T}\left|\left[\varphi_{l}\right]_{A}\left(x_{l}\right)\right|\left\|\operatorname{curl} \psi_{l}\right\|_{0, T} \\
& \leq c \sum_{T^{*} \subset \tilde{T}}\left\|e_{T^{*}}\right\|_{0, T^{*}}
\end{aligned}
$$

We return to (4.9). Since the number of triangles in $\tilde{T}$ only depends of the minimum angle, by using (4.10), (4.1) and (4.13) we obtain

$$
\begin{aligned}
\sum_{T \in \mathscr{T}}\left\|e_{T}\right\|_{0, T}^{2} & =\sum_{T \in \mathscr{T}}-\frac{1}{2}\left(\sum_{l \subset \partial T} \int_{l} \varphi J_{l}\right)=\sum_{T \in \mathscr{T} h}\left(\boldsymbol{\epsilon}_{h}, \operatorname{curl} \varphi\right)_{T} \\
& \leq \sum_{T \in \mathscr{T}}\left\|\boldsymbol{\epsilon}_{h}\right\|_{0, T}\|\operatorname{curl} \varphi\|_{0, T} \leq c\left\|\boldsymbol{\epsilon}_{h}\right\|_{0, \Omega}\|e\|_{0, \Omega}
\end{aligned}
$$

Which proves the theorem.

Remark 4.1 The lower bound in (4.6) is also of a local character. It can be easily shown that $\left\|e_{T}\right\|_{0, T} \leq c \eta_{T}$. So, because of (3.19), the statements in Remark 3.3 are also valid for this estimator. 


\section{Conclusions}

We introduce estimators for the Raviart-Thomas and Brezzi-Douglas-Marini spaces. These estimators are defined by means of evaluations of the residual, in the same way as those by Babuska-Miller (for divergence type) and Verfurth (for the mini-element discretization of the Stokes equations). These estimators are equivalent to the error, without assuming neither additional regularity of the solution nor any particular structure for the meshes.

In particular, for problems with corner singularities, the results are valid for those typical meshes obtained when adaptive mesh-refinement techniques are used. Actually, we can prove that we obtain local lower bounds for the error of the numerical solution. These results are analogous to Babuska-Miller's and Verfurth's ones for their problems and methods.

For the space $R T^{0}$, we define other estimator, wich is similar to Bank-Weiser's one, in the sense that is based on the solution of local problems.This estimator is also equivalent to the error under a saturation assumption. Once more, this result is similar to that obtained by Bank-Weiser for their estimator.

\section{References}

1. Babuska, I., Miller, A. (1987): A feedback Finite Element Method with a-posteriori error estimator: Part I. The Finite Element Method and some basic properties of the a posteriori error estimator. Comput. Meth. Appl. Mech. Engrg. 61, 1-40

2. Babuska, I., Rheinboldt, W.C. (1978): A posteriori error estimators in the finite element method. Internat. J. Numer. Meth. Engrg. 12, 1597-1615

3. BabuskaI., Rheinboldt, W.C. (1978): Error estimates for adaptive finite element computations. SIAM J. Numer. Anal. 15, 736-754

4. Bank, R., Weiser, A. (1985): Some a Posteriori error estimator for elliptic partial differential equations. Math. Comp. 44, 283-301

5. Bank, R., Welfert, B.D. (1991): A posteriori error estimators for the Stokes problem. SIAM J. Numer. Anal. 28, 591-623

6. Brezzi, F., Douglas, J., Marini, L. (1985): Two families of Mixed Finite Elements for 2-nd order elliptic problems. Numer. Math. 47, 217-235

7. Brezzi, F., Fortin, M. (1991): Mixed and Hybrid Finite Element Method. New York, Springer

8. Ciarlet, P. (1978): The Finite Element Method for elliptic problems. Amsterdam, North Holland

9. Douglas, J. Jr., Roberts, J.E. (1985): Global estimates for mixed methods for second order elliptic equations. Math. Comp. 44, 39-52

10. Raviart, P.A., Thomas, J. (1977): A mixed Finite Element Method for 2-nd order elliptic problems, Mathematical aspects of the Finite Elements Method. Lectures Notes in Math. 606, pp. 292 315. Berlin, Springer

11. Verfurth, R. (1989): A posteriori error estimators for the Stokes Equations. Numer. Math. 55, 309-325

12. Verfurth, R.: A posteriori error estimators and adaptive mesh-refinement techniques for the Navier-Stokes equations. In: M.D. Gunzburger, R.A. Nicolaides, eds., Incompressible CDFTrends and Advances, Cambridge, Cambridge University Press (to appear)

13. Verfurth, R.: A posteriori error estimator and adaptive mesh-refinement techniques (to appear)

This article was processed by the author using the $\mathrm{LAT}_{\mathrm{E}} \mathrm{X}$ style file pljour $1 m$ from Springer-Verlag. 